\title{
Toward a third generation of gravitational wave observatories
}

\author{
Michele Punturo • Harald Lück
}

Received: 14 September 2009 / Accepted: 6 May 2010

(C) Springer Science+Business Media, LLC 2010

\begin{abstract}
Large gravitational wave interferometric detectors, like Virgo and LIGO, demonstrated the capability to reach their design sensitivity, but to transform these machines into an effective observational instrument for gravitational wave astronomy a large improvement in sensitivity is required. Advanced detectors in the near future and third generation observatories in slightly more than one decade will open the possibility to perform gravitational wave astronomical observations from the Earth. An overview of the technological progress needed to realize a third generation observatory, like the Einstein Telescope (ET), and a possible evolution scenario are discussed in this paper.
\end{abstract}

Keywords Gravitational waves · Future detectors $\cdot$ Einstein telescope

\section{Introduction}

The first generation of interferometric gravitational wave (GW) detectors (GEO600 [1], LIGO [2], TAMA [3], Virgo [4]) demonstrated the effectiveness of the working

M. Punturo (ه)

Istituto Nazionale di Fisica Nucleare, Sezione di Perugia,

Via Pascoli, 06123 Perugia, Italy

e-mail: michele.punturo@pg.infn.it

H. Lück

Institut für Gravitationsphysik, Leibniz Universität Hannover,

Callinstr. 38, 30167 Hannover, Germany

e-mail: harald.lueck@aei.mpg.de 
principle [11] and in general the capability to reach their design sensitivity. The major detectors currently operative are enhanced versions of the first generation, with larger laser power and some technological improvements.

The design of the second generation of GW detectors ("Advanced LIGO" [5] and "Advanced Virgo" [6,7]) is relatively well established and the components are currently under realization. These detectors will show a sensitivity about a factor of ten better than the initial machines adopting technologies currently available, sometimes tested in reduced scale prototypes, but still to be implemented in full scale. According to the current models of gravitational wave sources the sensitivity of the advanced interferometers is expected to guarantee the detection of the signals generated by astro-physical sources. For example, at the nominal sensitivity of the advanced detectors, the expected detection rate of the gravitational wave signal generated by a binary system of coalescing neutron stars is about a few tens per year $[8,9]$. But apart from extremely rare events, the expected signal-to-noise ratio (SNR) of these detections obtained with the advanced detectors is too low for precise astronomical studies of the gravitational wave sources and to complement optical and X-ray observatories in the study of fundamental systems and processes in the Universe.

These considerations urged the gravitational wave community to start investigating a new generation of machines. With a largely improved sensitivity with respect to the advanced interferometers these new machines will open the era of routine gravitational wave astronomy. Since the first detection of a gravitational wave signal is expected to occur in the advanced interferometers, it is especially the understanding and enhancement of the observational aspects that the planning of the third generation must focus on.

To realize a third generation gravitational wave observatory with a significantly enhanced sensitivity, several limitations of the technologies adopted in the advanced interferometers must be circumvented and new solutions must be developed to reduce the fundamental and technical noises that will limit the next generation machines. In fact, in a third generation gravitational wave detector, there are additional requirements that make the achievement of the sensitivity targets very challenging; the first requirement is the improvement of the noise level in relation to the advanced detectors roughly by a factor of ten in the whole detection frequency band, but the second and more difficult requirement is to access the frequency region between 1 and $10 \mathrm{~Hz}$, excluded in the advanced detectors. The achievement of both these targets is too challenging to be requested from an improved seismic filtering system and alternative (or better additive) solutions must be found.

The purpose of this paper is to introduce these technological challenges (see Sect. 2), leaving the duty to detail the technical solutions to the dedicated, more specialized articles in this special issue; in Sect. 3 we will try to depict the possible evolution scenario and timelines to arrive at a third generation gravitational wave observatory, like the Einstein Telescope (ET) in Europe [10]. Because of the vastness of this subject, it is obvious that several technical issues, many possible technologies and important aspects, like simulation efforts and some R\&D needs, are missing in this paper. 


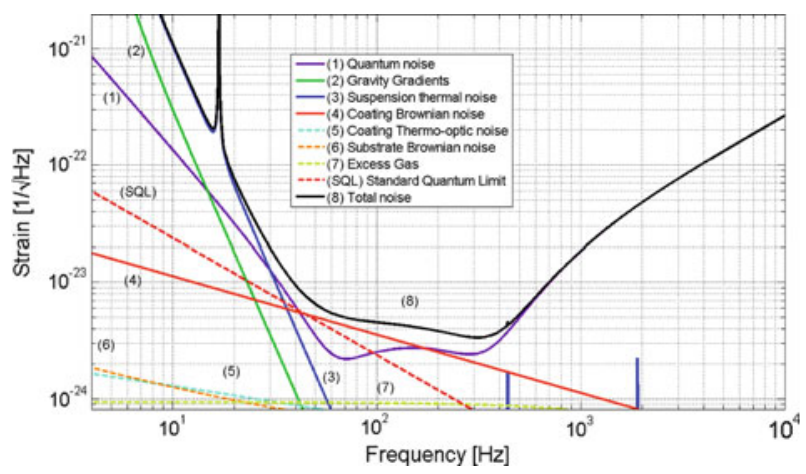

Fig. 1 Typical sensitivity of a second generation GW detector (for the sake of precision, it is the expected sensitivity of the advanced Virgo detector with $125 \mathrm{~W}$ of injected power); the sensitivity is dominated at very low frequency by the seismic noises (direct and through the gravity gradient coupling) that make the frequency range below $10 \mathrm{~Hz}$ unusable, at low and intermediate frequencies by the thermal noises (suspension and mirror) and by the quantum noise (radiation pressure component), at high frequencies by the quantum noise (shot noise component)

\section{Limits of the second generation detectors and technological challenges for the third generation}

The typical sensitivity of an advanced or second generation interferometric gravitational wave detector is shown in Fig. 1. If we consider only the fundamental noises, the sensitivity of these apparatuses will be dominated

- at very low frequency (below 4-5 Hz) by the seismic noise and the gravity gradient noise (see Sect. 2.1),

- in the 4-50 Hz by the thermal noise (see Sect. 2.2.1) of the optics suspension system and by the quantum noise, related to the radiation pressure (see Sect. 2.3.5) exerted on the suspended mirror by the photons in the main Fabry-Perot cavities,

- in the $40-300 \mathrm{~Hz}$ by the thermal noise of the suspended mirrors (mainly the coating contribution, see Sect. 2.2.2)

- and at higher frequency by the shot noise component of the quantum noise (see Sect. 2.3.2).

\subsection{Seismic noise}

Ground based interferometric gravitational wave detectors are and will be limited, in the very low frequency range, by the natural and anthropogenic vibration of the soil where the instrument is realised. The only way to fully eliminate this limiting source is to realise the detector in space, like it is planned for the LISA project $[12,13]$.

Seismic noise acts on the suspended test masses in two ways:

- through the suspension chain, shaking each stage according to its transfer function

- coupling the mass vibration in the soil layers, perturbed by the seismic waves, directly with the test masses displacement, via the mutual attraction force expressed 
by Newton's universal law of gravitation (so-called gravity gradient noise or Newtonian noise).

\subsubsection{Seismic noise filtering}

Although driven or affected by many natural and artificial factors, the seismic displacement spectral amplitude $x_{\text {seism }}$ can roughly be expressed, for frequencies larger than about $1 \mathrm{~Hz}$, by the simple equation:

$$
x_{\text {seism }}(f) \simeq \frac{A_{0}}{f^{2}}
$$

where the amplitude $A_{0}$ ranges between $10^{-8}$ and $10^{-6} \mathrm{~m} \cdot \mathrm{Hz}^{3 / 2}$, depending on the quietness of the detector site. Usually the seismic vibration is roughly isotropic and Eq. 1 is still valid in all the three directions. Obviously, in a gravitational wave detector, to have a reasonable sensitivity at a few Hertz, the seismic vibration should be sufficiently filtered by the suspension chain before it reaches the test masses. Currently this filtering system is realised through a chain of harmonic oscillators that filter the seismic vibration horizontally (inverted and "normal" pendulums) and vertically (blades). Virgo of all first generation detectors implemented the most sophisticated of such passive filtering systems and in fact Virgo is the interferometer that currently shows the best sensitivity below $40 \mathrm{~Hz}$. The so-called Super-Attenuator pushes the residual seismic noise below the thermal noise of a first generation detector like Virgo starting from about $4 \mathrm{~Hz}$ [14]. Recent evaluations [15], supported by direct measurements in the Virgo detector, demonstrated the full validity of the passive filtering design also considering the stringent requirements of a second generation gravitational wave detector like advanced Virgo, where the suspension thermal noise is reduced by an order of magnitude.

Passive filtering is not the only way to fight the seismic noise in a gravitational wave detector; in the advanced LIGO design, an active philosophy [16] is being implemented: in a chain of three sub-systems the displacement and the accelerations caused by the seismic noise are read through position and acceleration sensors and are actively and hierarchically suppressed through hydraulic and electro-magnetic actuators.

As anticipated in the Sect. 1, the widening and improving of the sensitivity, mainly at low frequency, in a third generation gravitational wave observatory, requires new solutions with respect to what is under design and realization in the advanced detectors. It is well known that underground sites are seismically quieter (i.e. see [17]) and the possibility to realise an underground gravitational wave detector has been analysed and selected by the LCGT [18] (Large Scale Cryogenic Gravitational wave Telescope) collaboration in Japan. The comparison between the seismic noise in the TAMA [19] site (Tokyo) and in the LISM [20] site (Kamioka mine, the prime candidate as an LCGT site) shows a reduction in the low-frequency region, by going underground, of a factor of 100 in terms of acceleration and by two to three orders of magnitude in displacement spectral amplitude. A corresponding and even larger noise reduction has been reached in the output of the LISM interferometer, due to the fact that going underground several other "technical" noises, induced by external disturbances like 
wind, scattered light or temperature fluctuations, are suppressed by the quietness of the site. It should be noted that this large improvement is also facilitated by the extreme difference between the two compared sites, the first one located in Tokyo, where a huge cultural noise is generated by the human activities, the second one far from human presence.

Hence, an underground site can offer the possibility to achieve the desired sensitivity improvement with respect to an advanced detector in the same frequency band. A preliminary investigation [15] on the compliance of the Virgo Super-Attenuator with the requirements of a third generation detector has shown a satisfactory behavior above $4 \mathrm{~Hz}$, meanwhile to access the $1-4 \mathrm{~Hz}$ frequency range major technical upgrades of the suspension system must be realised. These upgrades are currently objects of the design activity within the Einstein Telescope (ET) project, and some ideas are currently under discussion, like a very long $(\simeq 50 \mathrm{~m})$ suspension chain, with each element being about 7-10 $\mathrm{m}$ long (in order to push down the pendulum frequencies) and a mixed active-passive design.

\subsubsection{Gravity gradient noise}

In the gravitational wave interferometers, the suspended test masses are subject to the random gravitational forces generated by seismic noise and by moving massive bodies [21-23]. Obviously the importance of this disturbance depends on the seismic noise level and on the contribution of the other low-frequency noise sources to the noise budget; it is evident that the seismic filtering chain doesn't play any role for this noise source, since the gravitational force is a direct interaction between the suspended mirrors and the neighboring masses. In the first generation of gravitational wave detectors the gravity gradient noise does not play any role and in the advanced detectors (due to the improvement of the sensitivity at low frequencies) it starts approaching the overall noise level, but remaining negligible above $10 \mathrm{~Hz}$ (see Fig. 1).

In the third generation of gravitational wave detectors, the more stringent requirements in terms of sensitivity at low frequency enhance the importance of this noise source. The possible reduction of the seismic noise at an underground site opens new questions about the validity of the current noise modeling. Recent evaluations [24,25] have shown that it is not important to give a particular shape to the cavern hosting the detector apparatuses. The studies underlined the effectiveness (at least above 1-2 Hz) in the attenuation of the gravity gradient noise, going deeply underground ( $\gtrsim 100 \mathrm{~m})$. If that very low frequency range needs to be accessed to fulfil the science requirements of a third generation observatory, a major effort must be performed to complement the seismic attenuation with the subtraction of the residual gravity gradient noise through the signals extracted from a network of sensors located around the detector $[24,25]$.

\subsection{Thermal noise}

By thermal noise we indicate all those processes that modulate the optical path of the light in the interferometer coupling it to the Brownian fluctuation or to the 
stochastic fluctuation of the temperature field in the optical components. It is common to distinguish between the suspension thermal noise, affecting the position of the test masses (considered a rigid body) through the fluctuations of the suspension wires or fibres, and the mirror thermal noise, which is the overlap of all the fluctuation and dissipation processes occurring in the test masses and in the high reflectivity coatings.

\subsubsection{Suspension thermal noise}

To model and understand the thermal noise in the interferometers (in thermal equilibrium) two fundamental instruments are used: the equi-partition theorem, that relates the temperature of a system to its average energies, and the Fluctuation-Dissipation theorem [26], that relates the power spectrum of the fluctuations of a system $X(\omega)$ (in thermal equilibrium) to its dissipation processes, described by the mechanical impedance $Z(\omega)$.

$$
\langle X(\omega)\rangle^{2}=\frac{4 k_{B} T}{\omega^{2}} \mathcal{R}\left\{[Z(\omega)]^{-1}\right\}
$$

where $k_{B}$ is the Boltzmann constant and $T$ is the temperature of the system. The equipartition theorem tells how much thermal energy is present in a thermo-dynamical system, meanwhile the Fluctuation-Dissipation theorem describes how that energy is distributed in frequency. The strategies to reduce the thermal noise impact in the second generation gravitational wave detectors mainly rely on improvements of what has been applied in the initial machines, chiefly on the reduction of the dissipation in the suspension system, to concentrate all the fluctuation energy into the normal modes of the system, resulting in a low noise level off-resonance.

Already in the initial detectors particular attention has been devoted to the reduction of the dissipation in the suspension by selecting the right material for the suspension wires [27-30], by optimizing the clamping [31] and the mirror-to-wire contact [32] design. As evolution of the suspension firstly adopted in the GEO600 detector [33,34], the second generation of gravitational wave detectors will adopt the so-called monolithic [35] suspensions, fully realized in fused silica, which simultaneously reduces the clamping losses and minimizes the losses in the suspension fibres, because of the low mechanical dissipation of fused silica [36].

In addition to the minimization of the mechanical losses through the selection of the best materials and geometries another means for reducing the suspension thermal noise must be used in the third generation of gravitational wave detectors: the temperature. According to the equi-partition theorem, the temperature is directly proportional to the energy stored in each degree of freedom of the suspended system allowing to reduce the fluctuation amplitude by lowering the temperature, as indicated in Eq. 2. Furthermore, at low temperature, some materials show a suppression of the dissipation mechanisms.

Hence, cryogenics is one of the most appealing technologies to reduce the thermal noise of the optics suspension in a third generation gravitational wave detector. The first problem to be solved in a cryo-interferometer is how to cool down the test masses without introducing additional vibrations that spoil the very low frequency 
performances. New technologies are now available, for example based on cryo-cooling systems [37] that are actively damped to reduce the seismic vibration and that promise an easier achievement of low temperatures with less impact on the low frequency performance of the interferometer than previous cryo-coolers. Details of these technologies are reported in another article of this special issue edition [38], where the scheme of a vibration free cryostat is reported. A special design of the suspension system is needed to cool down the test mass without introducing additional vibration. Progress in channeling the heat transmission through a second, parallel seismic filtering system has been made in the ILIAS [39] project.

Even more important is the right choice of the material for the realisation of the last stage of the suspension system. The all-fused silica suspension, developed for the second generation gravitational wave detectors, cannot be used in cryogenics because of its poor thermal conductivity and because of a well known dissipation peak of that amorphous material at low temperature. In order to be a good candidate for a cryogenic suspension the material must exhibit a high thermal conductivity at the operation temperature chosen, to permit an efficient heat extraction (which is crucial, because of the relatively high heating power deposited in the test masses by the high light power stored in the interferometer cavities), a low mechanical dissipation angle (to reduce the Brownian thermal noise), a low thermal expansion coefficient (to minimise the thermo-elastic noise), and a good breaking strength (to safely support the test masses). Currently there are two candidate materials for this role: Sapphire and Silicon. Sapphire has been selected to realise the suspension fibres of LCGT both for its dissipation [40] and for its thermal conductivity properties [41]; Silicon, instead, has been preliminary studied within the ILIAS project and it has been found suitable to realise both suspension fibres [42] and ribbons [43]. However, it is matter of fact that currently only sapphire has been used to realize a full cryogenic suspension and the usage of Silicon still needs a successful R\&D activity.

\subsubsection{Mirror thermal noise}

The major limitation of the sensitivity of the second generation of gravitational wave detectors in the $40-200 \mathrm{~Hz}$ will be the thermal noise related to the high reflectivity dielectric coating applied to the main cavities mirrors. While the mirror substrate material chosen (like synthetic fused silica Heraeus Suprasil ${ }^{\circledR} 3001$ (http://optics. heraeus-quarzglas.com/en/productsapplications/productdetail_1938.aspx) or 3002) shows low mechanical dissipation, the high refraction component in the dielectric coating (Tantalum-pentoxide $\mathrm{Ta}_{2} \mathrm{O}_{5}$ ) shows high mechanical losses that dominate the dissipation of the test mass $[44,45]$.

There are several $R \& D$ activities, related to the advanced detector realization, that are attempting to reduce the contribution of the Tantalum-pentoxide, both by reducing its intrinsic losses by including Titanium dopants in the Tantalum layers [46] and by optimizing the amount of high dissipation material while keeping the same reflectivity [47]. The introduction of the titanium dopant permits to reduce the loss angle by $\approx 50 \%$ [46], with an important impact on the thermal noise level and detection distance (but not negligible increase of the optical absorption). The optimization of the Tantalum-pentoxide layers' total thickness seems to permit a reduction of the noise 
power spectral density by a factor of 0.83 boosting the instrument visibility distance by a $30 \%$ [50]. For maximum benefit both techniques can also be combined.

These studies on the coatings for the advanced detectors are very important also for the third generation of the gravitational wave detectors, but unfortunately we cannot expect a further reduction of the dissipation if a cryogenic-interferometer is realized. In fact, measurements performed to characterize the coatings for the LCGT detector have shown that the mechanical dissipation in a multi-layer Tantalum-pentoxide coating is rather independent of the temperature [48] and more recent measurements [49] have even shown a low temperature dissipation peak in a single-layer of $\mathrm{Ta}_{2} \mathrm{O}_{5}$ doped with $\mathrm{TiO}_{2}$.

Furthermore, because of the broad dissipation peak shown by fused silica at low temperature, in the cryogenic interferometers it is impossible to choose the low mechanical loss, low optical absorption substrates developed for the advanced detectors. Hence, as a result from the suspension development (see Sect. 2.2.1), currently the best material candidate to realize the test masses is Sapphire (selected in LCGT) and crystalline Silicon, which shows a very low mechanical dissipation angle (about 3-4 $\times 10^{-9}$ ) at low temperature. Sapphire is transparent to the standard wavelength adopted in the gravitational wave detectors $(1064 \mathrm{~nm})$. It shows relatively small thermal lensing [51] due to its large thermal conductivity at low temperature $\left(2330 \mathrm{~W} \cdot \mathrm{m}^{-1} \mathrm{~K}^{-1}\right.$ at $10 \mathrm{~K}$ ), but the high optical absorption (about $90 \mathrm{ppm} / \mathrm{cm}$ [52]) measured in the available substrate samples, constrains the interferometer design and limits the future light power increase in the main Fabry-Perot cavities. Future improvement in the optical properties of the Sapphire substrate could enhance the possibilities of this solution. Silicon shows a similar thermal conductivity $\left(1200 \mathrm{~W} \cdot \mathrm{m}^{-1} \mathrm{~K}^{-1}\right.$ at $\left.12.5 \mathrm{~K}\right)$, but it is transparent only at a longer wavelength $(\lambda \gtrsim 1500 \mathrm{~nm})$, where it shows an incredibly low absorption (about $3 \times 10^{-8} \mathrm{~cm}^{-1}$ at $1445 \mathrm{~nm}$ [53]), which requires to reconsider all optical and electro-optical component choices in the interferometer.

To solve the coating problem, either a new high refraction index material with a low dissipation at cryogenic temperature, suitable to realize the needed high reflectivity coating, is found or a completely different solution must be developed. A promising $\mathrm{R} \& \mathrm{D}$ activity [54] is devoted to produce high reflectivity mirrors with just one dissipative layer of dielectric coating material on the substrate or even without any additional layer, realizing the so-called resonant waveguide grating [55] by nano-structuring the surface of the Silicon substrate.

A list of the different thermal noise sources present in the test masses is reported in Table 1, where the crucial parameters, for each noise source, are given in the last column. It is evident that the beam size plays a crucial role in all those noise sources and this pushes the request to make the beam as large as possible. Because of the finite size of the substrates, the requirements in terms of diffraction losses and in terms of optical stability of the Fabry-Perot cavities, the beam size cannot grow infinetely and some other technique must be investigated. The potential in terms of thermal noise reduction of Fabry-Perot cavities with resonant beams with a flatter radial distribution of the light intensity [57] is well known; some attention has been drawn towards the possibility of using Laguerre-Gauss modes of orders higher than $(0,0)$ resonating in the Fabry-Perot cavities [58]. According to the most recent results [59], in the case of a cryogenic interferometer $(10 \mathrm{~K})$ adopting a Silicon mirror with a diameter of 
Table 1 Mirror thermal noise contributions, evaluated well below the first resonance

\begin{tabular}{|c|c|c|}
\hline Source & Spectral Density & Crucial Parameters \\
\hline Coating & & $T:$ Temperature \\
\hline $\begin{array}{l}\text { Brownian } \\
\text { Noise }\end{array}$ & $S(\omega)=\frac{4 k_{B} T\left(1-\sigma_{s}\right)^{2}}{\sqrt{\pi} \omega Y_{s}} \frac{\phi_{c}}{w}$ & $\begin{array}{l}w^{-1}:(\text { Beam waist })^{-1} \\
\phi_{c}: \text { Coating loss angle }\end{array}$ \\
\hline Substrate & & $T$ \\
\hline $\begin{array}{l}\text { Brownian } \\
\text { Noise }\end{array}$ & $S(\omega)=\frac{4 k_{B} T\left(1-\sigma_{s}\right)^{2}}{\sqrt{\pi} \omega Y_{s}} \frac{\phi_{s}}{w}$ & $\begin{array}{l}w^{-1} \\
\phi_{S}: \text { Substrate loss angle }\end{array}$ \\
\hline Substrate & $S(\omega)=\frac{8 k_{B} T^{2} \alpha_{S}\left(1+\sigma_{s}\right)^{2} w}{\sqrt{2 \pi} \kappa_{S}} J[\Omega]$ & $T^{2}$ \\
\hline Thermo-elastic & $\begin{array}{l}\Omega=\omega / \omega_{c}, \omega_{c}=\frac{4 \kappa}{\rho C w^{2}} \\
J[\Omega]=\sqrt{\frac{2}{\pi^{3}}} \int_{0}^{+\infty} d u \int_{-\infty}^{+\infty} d v \frac{u^{3} e^{-u^{2} / 2}}{\left(u^{2}+v^{2}\right)\left[\left(u^{2}+v^{2}\right)^{2}+\Omega^{2}\right]}\end{array}$ & $\begin{array}{l}\approx w^{-2} \\
\alpha_{s}^{2}: \text { Substr. th. exp. coeff. }\end{array}$ \\
\hline Coating & & $T^{2}$ \\
\hline Thermo-elastic & $S(\omega)=\frac{2 \sqrt{2} k_{B} T^{2}}{\pi \sqrt{\omega \kappa_{s} c_{s} \rho_{s}}} \frac{\left(\alpha_{c}-\overline{\alpha_{s}} \frac{\left\langle c_{c} \rho\right\rangle}{c_{S} \rho_{s}}\right)^{2}}{w^{2}} G(\omega)$ & $\begin{array}{l}w^{-2} \\
\alpha_{c}^{2}: \text { Coat. th. exp. coeff. }\end{array}$ \\
\hline Coating & & $T^{2}$ \\
\hline $\begin{array}{l}\text { Thermo- } \\
\text { refractive }\end{array}$ & $S(\omega)=\frac{2 \sqrt{2} k_{B} \cdot T^{2}}{\pi \sqrt{\omega \cdot \rho_{c} \cdot c_{C} \cdot \kappa_{c}}} \frac{\beta_{c}^{2}}{w^{2}}$ & $\begin{array}{l}w^{-2} \\
\beta_{c}^{2}: \text { Coat. refr. index th. grad. }\end{array}$ \\
\hline
\end{tabular}

$\sigma$ Poisson ratio, $Y$ Young's modulus, $\phi$ loss angle, $\rho$ density, $c$ specific heat (per mass unit), $w$ beam waist on the mirror, $\alpha$ thermal expansion coefficient, $\kappa$ thermal conductivity, $\beta$ refraction index " $n$ " thermal gradient $\frac{d n}{d T}, \lambda$ laser wavelength. Index $s$ indicates a "substrate" property, $c$ a "coating" property. For a more exhaustive description of the formulae and symbols in this table see [56]

$62 \mathrm{~cm}$ and a thickness of $30 \mathrm{~cm}$, resonating a $(3,3)$ Laguerre-Gauss mode with a beam waist radius of $7.2 \mathrm{~cm}$ it is possible to suppress the cumulative mirror thermal noise by a factor 1.71 with respect to a Gaussian beam with a waist size if $11.9 \mathrm{~cm}$ (beam sizes selected to give diffraction losses of $1 \mathrm{ppm}$ ). These numbers show that it is important to push the development of such techniques for minimizing the thermal noise contributions to the overall noise budget.

\subsection{Quantum noise}

\subsubsection{The origin of quantum noise}

The interferometric measurement of gravitational waves relies on the conversion of a differential length change of the interferometer arms into an intensity change of the light at the output of the interferometer. All first generation (initial) and second generation (advanced) interferometric gravitational-wave detectors are based on the Michelson principle (see Fig. 2). In all of them the operating point is chosen such that all field components symmetrically present (in amplitude and phase) in both interferometer arms (like the laser carrier light), returning from both arms to the beam 

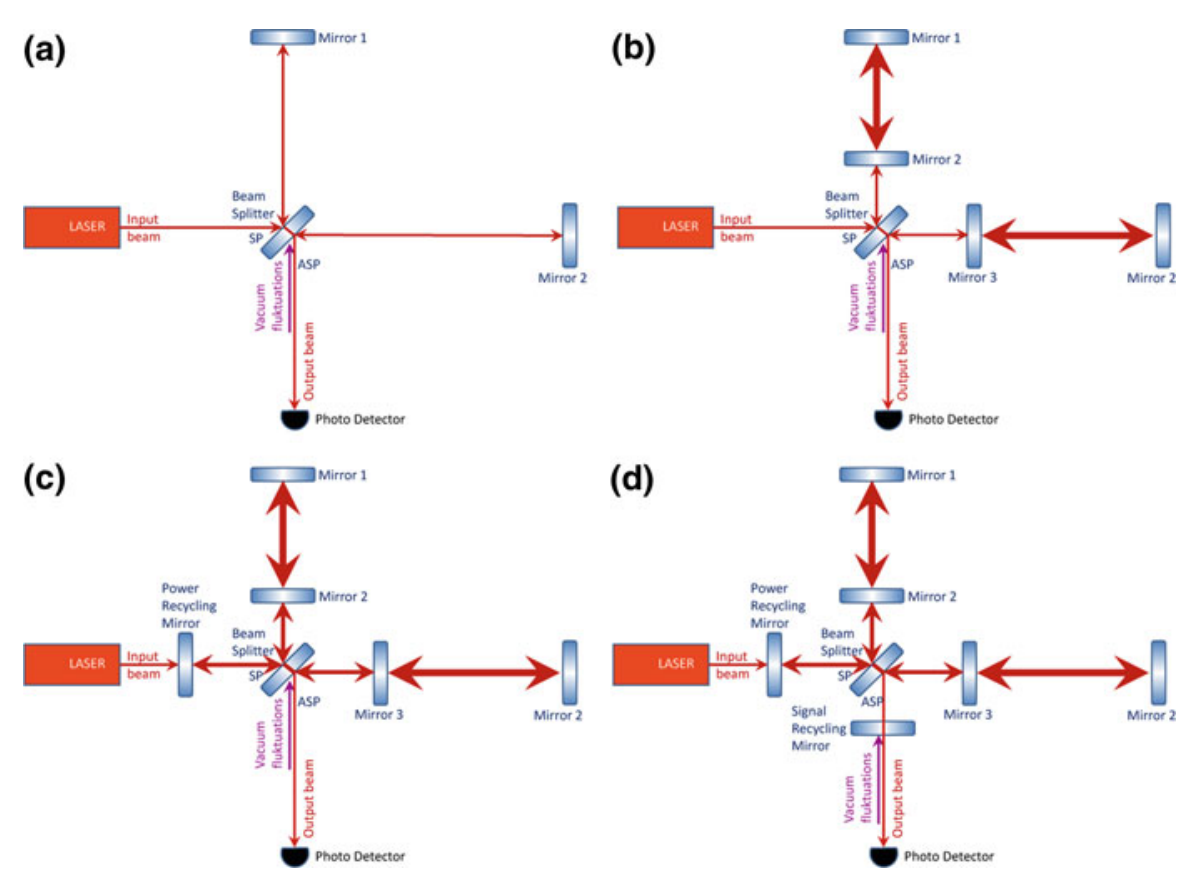

Fig. 2 Schematic views of Michelson interferometer layouts. a Simple Michelson interferometer without any resonant power enhancement; b Michelson Interferometer with Fabry-Perot arm cavities for resonant power enhancement; c Michelson interferometer with arm cavities and Power Recycling; d Michelson interferometer with arm cavities and dual recycling. The symmetric and anti-symmetric ports of the beam splitter are labelled 'SP' and 'ASP', respectively

splitter, constructively interfere towards the input (symmetric) port and destructively interfere towards the output (anti-symmetric) port. A GW, lengthening one arm while shortening the other, produces anti-symmetric signal sidebands in both arms, which, returning to the beam splitter, constructively interfere at the anti-symmetric port, the output port. Due to phase relations (resulting from energy conservation) at the beam splitter [60], fields entering the interferometer from the output port, get split into the two interferometer arms with a phase shift of $+/-\pi$, w.r.t. the light entering from the symmetric port. Hence these fluctuations entering the output port cause anti-symmetric field components in the interferometer arms, which, detected at the anti-symmetric port, cannot be distinguished from a GW signal. Therefore it is mostly fluctuating fields entering the output port that we have to worry about.

Quantum Noise in interferometric gravitational-wave detectors arises from the quantization of the electro-magnetic field. According to Quantum Mechanics the lowest energy state of the quantized electro-magnetic field (also called zero-point field or vacuum state) has a fluctuating amplitude equivalent to a mean energy of $\hbar \omega / 2$ per mode of the field, distributed over both quadratures, amplitude and phase. The level of fluctuations can be traded between the quadratures, as only the product is limited by Heisenberg's uncertainty principle. These omnipresent fluctuations can enter the interferometer through the output port, anti-symmetrically add to the light field in the 
interferometer arms and cause noise inside the interferometer and in the output field: the Quantum Noise. A comprehensive description of Quantum Noise and means to fight it can be found in [61] and references cited within.

The quantum noise usually arises in two forms: shot noise and radiation pressure noise. Shot noise is the intensity Quantum Noise on the photo detector. Phase noise of the vacuum fluctuations, entering the interferometer through the output port, gets converted into intensity fluctuations of the light returning to the output port, which is then measured by the photo-detector. Without the vacuum fluctuations from the output port the signal from the interferometer would only show negligible shot noise on the output beam. Radiation pressure noise results from the impulse transfer of the photons upon reflection from a test mass. The (differential) quantum fluctuations in the power of the beams impinging on the mirrors leads to real mirror displacements having the same effect as a gravitational wave.

The resulting strain noise spectral density for a simple Michelson interferometer in the frequency range where the mirrors behave like free masses is given by:

$$
\begin{aligned}
S_{h} & =(1 / \kappa+\kappa) \frac{h_{\mathrm{SQL}}^{2}}{2} \\
\text { with } \quad \kappa & =\frac{4 I_{0} \omega_{0}}{c^{2} M \Omega^{2}} \text { and } h_{\mathrm{SQL}}=\sqrt{\frac{4 \hbar}{M \Omega^{2} L^{2}}}
\end{aligned}
$$

where $\omega$ is the angular frequency of the laser light, $\Omega$ the gravitational wave signal frequency, $L$ the arm length, $c$ the speed of light, $M$ the mirror masses, and $I_{0}$ the laser power. The vacuum fluctuations are assumed to be equally distributed on amplitude and phase noise.

In Eq. 3 the $1 / \kappa$ term represents the shot noise at the readout while the $\kappa$ term stands for the radiation pressure effect. The optimal sensitivity that can be reached for a given frequency by trading shot noise versus radiation pressure by chosing the best light power is called the Standard Quantum Limit (SQL).

While the amount of the vacuum fluctuations entering the interferometer through the output port is constant, the amount of carrier light in the interferometer arms can be changed. This results in a shot noise level on the photo-detector, which is proportional to the amplitude of the light leaving the interferometer output. The gravitational-wave signal on the other hand is proportional to the light power inside the interferometer, which translates into a proportionality of the light power on the photo-detector, as long as no other power dependent processes introduce additional noise. Hence the Signal-to-(Shot)Noise Ratio scales with the square-root of the light power in the interferometer.

\subsubsection{Increasing the light power}

So one solution for increasing the sensitivity (for frequencies where shot noise dominates) is to increase the light power inside the interferometer arms. High power in the interferometer arms is achieved by three different means: 
- Arm cavities

- High power lasers

- Power recycling

Arm cavities Placing Fabry-Perot cavities in the interferometer arms (see Fig. 2b) resonantly enhances the light in the individual arms and with it the signals created by the GWs. The amount of power enhancement is limited by the fact that the linewidth of the optical resonator decreases with increasing build-up until relevant parts of the frequency spectrum fall outside of the resonance of the cavity and the GW signals do not get simultaneously enhanced with the carrier light any more. The low transmission of the input mirror then causes a reduction of the signal amplitude for high frequencies signals at the output port, i.e. a reduced sensitivity. The possible power enhancement in the interferometer arms is hence a compromise between a low relative shot noise level and bandwidth.

High power lasers The most obvious way to increase the light power inside the interferometer is to use a powerful laser. All first and second generation interferometric GW detectors use lasers with a wavelength of $1064 \mathrm{~nm}$. During the construction and operation of the first generation the available laser power increased from about 10$20 \mathrm{~W}$ in the first generation to $200 \mathrm{~W}$ available for the second generation. According to current plans solid state $1 \mathrm{~kW}$ lasers will be available for the third generation. The use of high laser power gives rise to thermal problems with the light absorbed in auxiliary optics like modulators, Faraday rotators or polarizers. The absorbed light will lead to a local temperature increase which through the temperature dependence of the refractive index will lead to thermal lensing. This in turn can lead to a reduced quality of mode matching to the optical resonators. Even the high quality main optics available today may exhibit too much absorption to cope with MW levels of light power. Although the absorption levels of fused silica have been cut down in the past to a level of $0.25 \mathrm{ppm} / \mathrm{cm}$ for bulk absorption and to the sub-ppm level for coating absorption, thermal problems will still pose a problem for the second generation of detectors. Elaborate thermal compensation systems are needed to compensate the effect.

The need to achieve low absorption combined with good mechanical performance at low temperatures (see Sect. 2.2.2 and [38]) will require to select a new material for the test masses. The physical properties of Silicon make it a good candidate. It exhibits good mechanical properties even at low temperatures [62-66] and promises extremely low absorption values at the wavelength of $1550 \mathrm{~nm}$ [53]. As the absorption at the standard laser wavelength, $1064 \mathrm{~nm}$, is high, new high power lasers are needed for this wavelength. High power fibre lasers are currently being developed but have not yet reached the performance level of solid state lasers. A current overview of available laser sources and prospects for the third generation of interferometric gravitational-wave detectors is given in [67].

Power recycling The carrier light as well as all common fluctuations returning from the interferometer arms constructively interfere towards the input port where they can be reflected with a mirror located in the input, called Power Recycling mirror (see Fig. 2c). The interferometer together with this mirror then forms an optical resonator 
Table 2 Typical light powers and mirror masses for different generations of GW interferometers

\begin{tabular}{llll}
\hline Detector generation & First & Second & Third \\
\hline Laser power & $10 \mathrm{~W}$ & $200 \mathrm{~W}$ & $1 \mathrm{~kW}$ \\
Light power @ beam splitter & $200 \mathrm{~W}$ & $2 \mathrm{~kW}$ & $20 \mathrm{~kW}$ \\
Light power inside ifo arms & $15 \mathrm{~kW}$ & $800 \mathrm{~kW}$ & $3 \mathrm{MW}$ \\
Mirror mass & $10 \mathrm{~kg}$ & $40 \mathrm{~kg}$ & $200 \mathrm{~kg}$ \\
\hline
\end{tabular}

where the light power can resonantly be enhanced. This technique is called Power Recycling and essentially has the same effect as directly increasing the laser power injected into the interferometer. All detectors of the first and second generation use and will use Power Recycling. With a laser power of about $200 \mathrm{~W}$ the second generation will reach power levels inside the arm cavities of around $800 \mathrm{~kW}$. The third generation is aiming at a light power in the interferometer arms in the few Megawatt range. With the promising prospect of $1 \mathrm{~kW}$ high-power-lasers available for the third generation there will be no need to increase the power recycling factor beyond what is foreseen for the second generation.

Approximate numbers for power requirements for the first to the third generation are given in the upper part of Table 2.

\subsubsection{Signal recycling}

The second generation of interferometric gravitational-wave detectors (in this sense treating GEO600 as a second generation instrument) uses an additional technique to lower the relative shot noise: Signal Recycling. The signals being generated by the GW (with a phase difference of $\pi$ ) in the interferometer arms constructively interfere towards the output port. If these signals are sent back towards the interferometer with a recycling mirror (see Fig. 2d) they get reflected back to the output port by the interferometer, similar to the laser power being reflected back to the input port. Together with the interferometer this mirror forms an optical resonator, the Signal Recycling cavity. By microscopic adjustment of the position of the Signal Recycling mirror the resonance of this cavity can be tuned to any desired frequency. Depending on this tuning the bandwidth of the interferometer can either be narrowed (called Signal Recycling in the case where the carrier frequency is close to a resonance) or widened (called Resonant Sideband Extraction in the anti resonant case). The tuning changes the resonance conditions of the fields in the signal recycling cavity and with it the phase relation between the signal sidebands and the carrier at the output port and inside the interferometer arms. Signal Recycling enhances the GW Signal being generated inside the optical cavity but does not enhance the fluctuations entering the cavity from the output port.

Beside the optical resonance, detuned Signal Recycling produces another optomechanical resonance, typically at lower frequencies (see Fig. 1), which gives an increase in sensitivity and, through the correlations that it introduces between amplitude and phase fluctuations, even allows to surpass the Standard Quantum Limit $[68,69]$. Already in the advanced detectors this additional optomechanical resonance 
lets the Quantum Noise fall below the Standard Quantum Limit, as can be seen in Fig. 1.

\subsubsection{Squeezing}

As indicated above the fluctuations in the different quadratures of the field entering the output port can be traded against each other, still fulfilling the requirements of Heisenberg's uncertainty relation. If such a squeezed state, where the phase noise is lowered at the cost of the amplitude noise, is injected into the output port, the shot noise of the light registered by the photo-detector will be lowered. At the same time the radiation pressure contribution will increase. By appropriately rotating the phase angle of the squeezing as a function of frequency, which can be achieved by sending the squeezed state through filter-cavities [70-72], the shot noise (at high frequencies) and the radiation pressure noise (at low frequencies) can be simultaneously reduced. In case of using squeezing together with a detuned interferometer, i.e. not tuned to zero frequency, which means that the laser carrier frequency is not resonant in the Signal Recycling cavitiy, the rotation of the squeezing ellipse with respect to the light coming from the interferometer (due to the dispersion of the Signal Recycling cavity) causes an increased amount of Quantum Noise outside of the Signal Recycling resonance due to the anti-squeezed quadrature of the injected squeezing [69]. Instead of filtering the squeezed state similar to fighting the radiation pressure noise, as mentioned above, this effect can also be avoided by using twin Signal Recycling [73]. In addition twin Signal Recycling resonantly enhances both signal sidebands, at positive and negative frequencies.

Squeezing is already being implemented in the current generation of interferometric gravitational-wave detectors in the case of GEO-600 [74], aiming at a squeezing level of $6 \mathrm{~dB}$ over the entire frequency range of interest. $6 \mathrm{~dB}$ effective squeezing at the photo-detector lowers the shot noise by a factor of two corresponding to a light power increase of a factor of 4 . An overall gain from squeezing of about $10 \mathrm{~dB}$, i.e. a factor of 3 in noise amplitude spectral density, for the third generation presently seems realistic.

\subsubsection{Radiation pressure noise}

Whereas increasing the power in the interferometer lowers the relative readout noise, i.e. the shot noise, it increases the radiation pressure noise. The impulse transfer of the photons onto the mirrors upon reflection causes a force acting onto the mirrors, the radiation pressure $p: p=2 I / c$, where $I$ is the light power and $c$ the speed of light. The amplitude fluctuations on the light this way get converted into mirror motion causing the so called radiation pressure noise. The mechanical susceptibility of a free mass (or a pendulum mass well above its resonance frequency) to a force is $(M \Omega)^{-2}$, were $M$ is the mass of the test mirror and $\Omega$ the Fourier frequency of interest. Radiation pressure therefore is more important at low frequencies, which also shows in Eq. 3. The worsening of the Quantum Noise at frequencies below about $20 \mathrm{~Hz}$ shown in Fig. 1 is due to this effect. 
Increasing the mirror mass decreases the mechanical susceptibility and hence cuts down the effect of radiation pressure on test mass movement. Consequently the mirror mass will increase from the first to the third generation as indicated in Table 2.

Reducing the differential amplitude fluctuations in the interferometer arms by using squeezed states replacing the vacuum fluctuations entering the output port can further lower the radiation pressure effect.

\subsubsection{Parametric instabilities}

The use of high power in the resonant Fabry-Perot cavities may show a crucial drawback: parametric instabilities [75]. This is a process in which the coupling between optical modes of resonant cavities and acoustic modes in the cavity mirror substrates creates a combined resonance which when excited would spoil the cavity's performance, up-to the unlock of the cavity. These parametric instabilities may be a serious problem for the advanced interferometers [76,77]. Studies for LCGT [78] have shown that the problem is less severe in this interferometer because of the different geometry and materials of the mirrors, since the radius of curvature of the mirror and the sound velocity at the operative temperature play a relevant role in determining the number of instable modes.

Hence, for a third generation gravitational wave detector, the evaluation of the relevance of the parametric instabilities issue strongly depends on the selected design options and for this reason the current ET design parameters are hereafter used to describe the possible situation. It ha been computed [79] that the number of the instable modes of the ET cavity is a few hundreds times larger than that of the LCGT cavity and it is probable that a methods to suppress the parametric instabilities is needed. The reduction of the mechanical $Q$ factor of elastic modes is a promising method currently investigated in advanced detectors and LCGT: a mechanical loss is deliberately added to the mirror, in order to damp the elastic modes. If the mechanical loss is concentrated far away from the optical beam, it will not significantly contribute to the mirror thermal noise. A possible implementation of this solution is realized by coating the barrel surface with a dissipative layer of $\mathrm{Ta}_{2} \mathrm{O}_{5}$. This method seems promising for ET if a Sapphire substrate is used, meanwhile in case of Silicon substrate, the excess of thermal noise could affect the expected sensitivity. These studies suggest that the parametric instabilities may be an issue for the third generation of gravitational wave observatories, but there are possible technical solutions that will evolve with the progress of dedicated R \& D.

\section{Scenarios for the third generation GW observatories}

In this section we will describe a possible evolution scenario that should lead us to the third generation of gravitational wave detectors. Obviously we will describe the most probable options based on the currently known technologies. For this reason we will neglect (although they may be scientifically interesting) some new solutions like atom-interferometers [80] and also QND techniques [81] which hold a high potential for lowering quantum noise but still need to be experimentally proven. 


\subsection{Single detector or multi-detector observatory}

As described in the previous section, to realise a third generation gravitational wave detector, the technologies currently operative in the initial and planned for the advanced detectors must be stressed and new solutions must be adopted. Although the R\&D advances could lead to a completely different result, a third generation gravitational wave detector could be based on the following technologies

- long arms, probably about $10 \mathrm{~km}$ long, to enhance the sensitivity to the dimensionless space-time strain $h$;

- underground site, to suppress the seismic and gravity gradient noises;

- long seismic filtering chains, to push the low frequency limit toward $1 \mathrm{~Hz}$;

- cryogenic test masses, to suppress suspension and mirror thermal noises;

- large and flat beams, to suppress thermal noise and mitigate the mirror thermal lensing;

- high power laser (about $1 \mathrm{~kW}$ ), high finesse Fabry-Perot cavities, high power recycling factor, signal recycling and squeezed light state injection, to suppress the quantum (shot) noise;

- heavy test masses and filtered squeezed state injection, to suppress radiation pressure noise.

A first evaluation of the potential of a similar detector has been performed within the ET project, considering only conventional technologies, defined by the authors of [82], fulfilling at least one of the following two criteria:

- The technology was already successfully demonstrated on prototypes, such as the injection of squeezed light into a Michelson interferometer or interferometry with cryogenic mirrors.

- The technology is an up-scaling of currently used technology without any change of the fundamental physics involved, for instance using suspensions of $50 \mathrm{~m}$ length.

Targeting for a wide-band detector, the sensitivity (named ET-B) of an underground, long suspension, cryogenic, signal and power recycled single Fabry-Perot enhanced Michelson detector has been evaluated (see Table 1 in [82]) and the resulting sensitivity is reported in Fig. 3.

In this evaluation, the cross-compatibility between the different technologies has been neglected, but the technological difficulty to realise a similar machine is evident. Let us have a look at an example of conflicting requirements:

- to minimize the shot noise, in the high frequency range, a large energy must be stored in the main cavities (3 MW in [82]); although a Silicon test mass could have negligible absorption, the high reflectivity coating could show an absorption of $0.1-0.5 \mathrm{ppm}$, causing a power deposition of about $0.3-1.5 \mathrm{~W}$ in the mirrors.

- the improvement of the low frequency noises requires long suspensions (seismic and thermal noise minimisation), thin suspension fibres (minimisation of the thermal noise) and cryogenic temperature (minimisation of the thermal noise). Although, thanks to the large thermal conductivity of the substrate material at low temperature, the usage of a cryogenic interferometer reduces the thermal lensing issues, it requires short and thick suspension fibres to extract the heat from the test masses. 


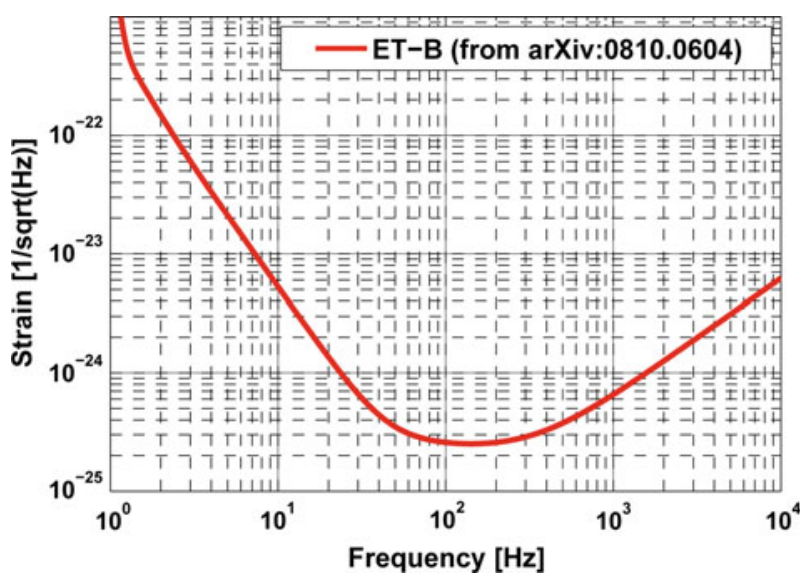

Fig. 3 Sensitivity of an underground, long suspension, cryogenic, signal and power recycled single third generation gravitational wave detector (see Table 1 in [82])

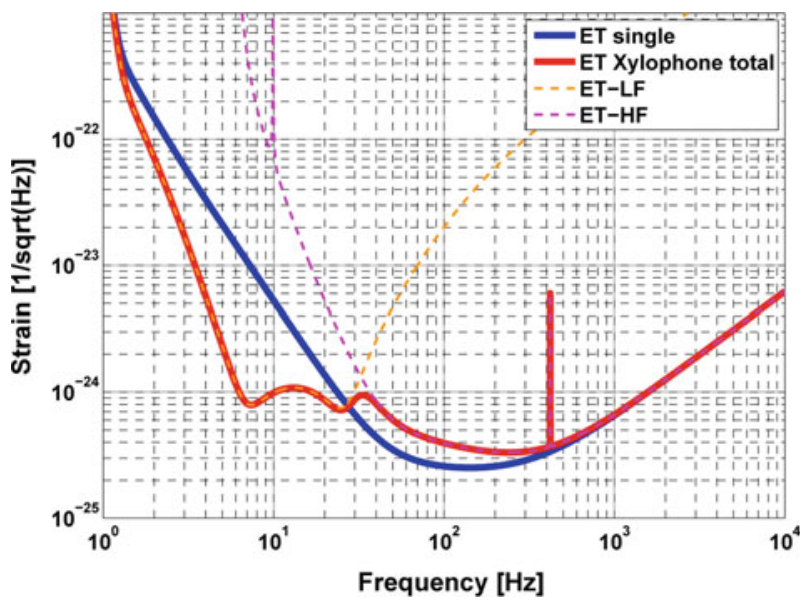

Fig. 4 Sensitivity of a third generation gravitational wave observatory implemented by two frequencyspecialised detectors [85]

For this reason the possibility to realise a wide-band third generation observatory, combining two (or more) detectors, specialised on different frequency bands, has been evaluated in [85]. In this case the output of a low-frequency-specialised detector is combined with the output of a high-frequency machine. The former one could be realized at an underground site by a cryogenic interferometer, long suspensions, but limited optical power, meanwhile the high frequency interferometer could be essentially a long arm advanced detector, implementing squeezed light states, a very high power laser and large test masses. This so-called Xylophone philosophy [83,84] essentially uncouples the technological requirements of a high power interferometer from the requirements of a cryogenic detector; the sensitivity of a similar observatory, evaluated in [85] for the ET design study, is reported in Fig. 4. 


\subsection{Detector geometry}

All the currently active gravitational wave detectors are L-shaped, with orthogonal arms; although this geometry maximises the sensitivity of the single detector with respect to the arm length, other geometries are possible. In particular, triangularshaped detectors have been proposed in the past [86,87] and also the LISA geometry is triangular. A detailed analysis of the benefits and drawbacks of a triangular-shaped third generation gravitational wave observatory is reported in [88] and here we report only the conclusions.

Co-located interferometers could be extremely useful to extract additional information from the gravitational wave observation; for example, two L-shaped detectors, forming a $45^{\circ}$ angle, could fully resolve the two polarisation amplitudes of the incoming wave. Three co-located interferometers, rotated by an arbitrary angle, through the virtual interferometry $[89,90]$ technique, could do the same, supplying additional benefits like null-stream channels and redundancy. Obviously in an underground site, the realisation of a similar cluster of orthogonal L-shaped detectors is practically impossible, because the huge cost of the infrastructures (several tunnels to accommodate the arms, several caverns to realise the central and end stations), but if the angle between the two arms of each detector is reduced to $60^{\circ}$, three detectors can be accommodated in a triangular-shaped underground site, minimizing the total length of tunnels, probably the number of caverns and recovering a sensitivity equivalent to two sets of orthogonal L-shaped double detectors, rotated by $45^{\circ}$ (see Fig. B1 of [88]).

For these reasons and because of the relevant role that the cost of the infrastructures will play in a third generation gravitational wave underground observatory, the selection of the geometry will probably be driven by the selection of the site and not vice versa: if a site that can accommodate a triangular observatory is found, the triple co-located interferometers may be the best choice, otherwise the two sets of orthogonal L-shaped double detectors will be more appealing.

\subsection{Network of detectors}

So far we assumed that a single site facility forms the third generation observatory. Depending on the astrophysical goals, the directionality of a detector could be of crucial importance, playing a role even larger than the sensitivity. This can be the case if a gravitational wave source needs to be spotted in a multitude of electro-magnetic sources.

For example, currently a network of at least three well separated detectors is needed to reconstruct the direction in the sky of a coalescing binary star system, the most promising gravitational wave source. In fact, to fully resolve a gravitational wave source, five parameters need to be reconstructed (right ascension $\alpha$, Declination $\delta$, Polarisation angle $\Psi$, Luminosity distance $D_{L}$, Orientation of the binary with regard to the line of sight $i$ ). In the so-called restricted post-Newtonian approximation [91], the antenna response $h(t)$ of a single detector is a linear combination of the two polarisations $\left(H_{+}, H_{\times}\right)$of the gravitational wave $\left[h(t)=A_{+}\left(\alpha, \delta, \Psi, D_{L}, i\right) H_{+}+\right.$ $\left.A_{\times}\left(\alpha, \delta, \Psi, D_{L}, i\right) H_{\times}\right]$and from a set of three detectors three amplitudes and two 
time-delays can be measured, yielding enough information to reconstruct the five parameters.

Due to the improved sensitivity, a third generation gravitational wave observatory could gain a lot in directionality for this kind of signal. If the signal source is a coalescing binary system, composed of two massive stars having unequal masses, for some inclination angle, the higher harmonics, due to higher multipole moments characterizing the source, play a relevant role in the waveform determination [91,92]. Since it is expected to detect the waveform with large SNR (up to several tens at $3 \mathrm{Gpc}$, as shown, for example, in Fig. 3 of [93]), higher harmonics of the gravitational wave signal are revealed with enough SNR to be used independently to reconstruct the source parameters; two detectors at the same site, in principle, allow the measurement of two amplitudes, the polarisation, inclination angle and the ratio $A_{+} / A_{\times}$, fully resolving the source. In practice, because of the limited accuracy, it may be advantageous to have two or more detection facilities spaced as widely as possible. Although only one third-generation project is being studied now, there is hope that other projects, e.g. a LIGO successor, will follow, resulting in a worldwide network of third generation detectors forming one big observatory. Using two distant detectors, the five parameters needed to reconstruct the source direction can be extracted from the four amplitudes from two sites and the single time delay.

For the detection of the stochastic GW background multiple co-located detectors would be best suited. The distance should be big enough to rule out common environmental noise sources but close enough that stochastic background noise still shows up coherently in the detectors.

\subsection{Timelines}

The evolution toward the third generation of gravitational wave detectors has been, is and will be a long path. Currently the main effort is made in Europe and only the European scenario will be depicted.

Past activities The first real attempt, in Europe, to have a design study of a third generation gravitational wave detector has been performed by the GEO600 and Virgo scientific communities in March 2004, submitting a proposal named "European Gravitational waves advanced Observatory design" (EGO) to the European Commission (Framework Programme 6-FP6). Although unsuccessful, this proposal highlighted the need of long preparation and coordination activities to arrive at a more successful trial. This coordination effort, in Europe, has been performed in the Networking activity N5 (http://www.ego-gw.it/ILIAS-GW/) within the ILIAS project (http://www.ilias. cea.fr/), supported by the European Commission (FP6) and it has also been pushed by the European Science Foundation (2005 ESF Exploratory Workshop (Toward a third generation European Gravitational Wave Observatory, http://esf-gw.pg.infn.it/).

Present status In May 2007 the GEO600 and Virgo collaborations responded to a call within the Framework Programme 7 (FP7) and submitted a proposal for a conceptual design study of a third generation gravitational wave observatory, named Einstein 


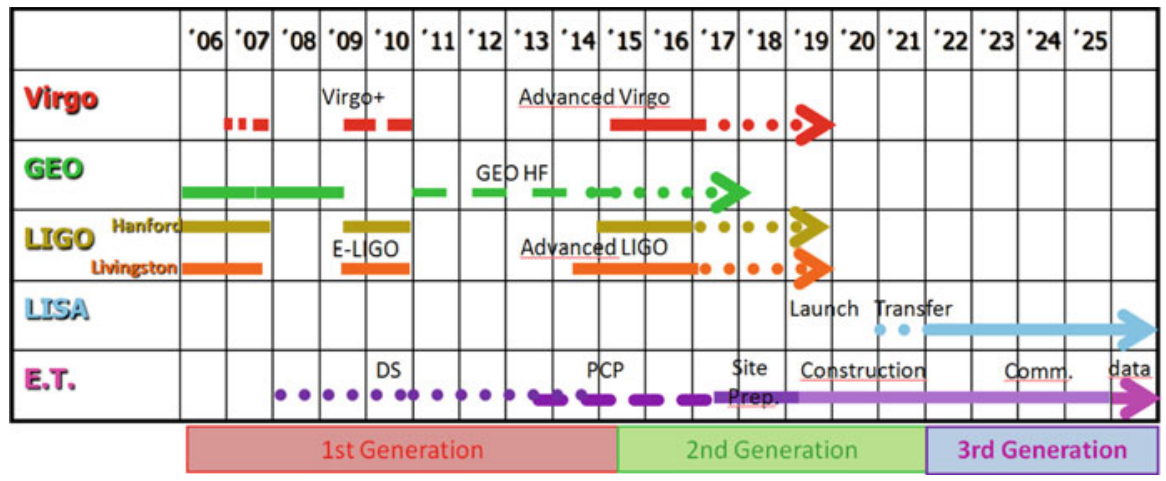

Fig. 5 Possible roadmap for the evolution of some of the gravitational wave detectors in the World. In the last line the expected evolution of the third generation observatory ET (Einstein Telescope) is shown. The time evolution of the Japanese interferometers (TAMA, LCGT, DECIGO) and of the other projects like ACIGA in Australia are not reported

Telescope (ET) [10] to the European Commission. This proposal was approved and funded for three years, starting from May 2008. The major goal of the ET project is to deliver a conceptual design for such a facility, investigating the technological feasibility, the science targets, the site requirements and prepare a costing draft for the infrastructure. The importance of the gravitational wave research and of the ET infrastructure has been recognised by the astro-particle community in Europe, who included ET in the list of the seven major future research infrastructures in Europe and in the ASPERA roadmap (http://www.aspera-eu.org/); the worldwide gravitational wave community accentuated the importance of ET in the GWIC roadmap (http:// gwic.ligo.org/roadldiscretionary-map/).

Future Although the efforts and the attention of the worldwide gravitational wave scientific community are currently focused on the realisation of the advanced detectors (advanced LIGO, advanced Virgo and LCGT), the activities devoted to the third generation must continue with increasing speed, because there is still a long path ahead. In Fig. 5 the expected evolution of the gravitational wave detectors in the World is reported. The last line of the table shows the long path still in front of the European project ET. After the current conceptual design study phase, a preparatory phase is expected to be necessary to better define the technological details, and the legal and organizational issues. The effective start of the construction (2018-2019) is expected to occur after the first detection of gravitational waves, which is reckoned to happen within at most one year after the advanced detectors will have reached their nominal sensitivity. The construction and commissioning of a third generation gravitational wave detector cannot be much shorter than what has been necessary for bringing the first generation of interferometers into operation and hence a period of about 6-7 years of intense activity is estimated to be needed before collecting the first science data with the Einstein Telescope ET.

Acknowledgments This work has been performed with the support of the European Commission under the Framework Programme 7 (FP7) "Capacities", project Einstein Telescope design study (Grant Agreement 211743), http://www.et--gw.eu/. 


\section{References}

1. Grote, H., et al.: Class. Quantum Grav. 25, 114043 (2008)

2. Abbott, B.P., et al.: Rep. Prog. Phys. 72, 076901 (2009)

3. Arai, K., et al.: J. Phys. Conf. Ser. 120, 032010 (2008)

4. Acernese, F., et al.: Class. Quantum Grav. 25, 114045 (2008)

5. Harry, G.M., LIGO Scientific Collaboration.: Advanced ligo: the next generation of gravitational wave detectors. Class. Quantum Grav. 27(8), 084006 (2010)

6. Flaminio, R., et al.: Advanced Virgo White Paper, VIRNOTDIR1390304 (2005)

7. Acernese, F., et al.: Advanced Virgo Baseline Design, VIR027A09 (2009)

8. Abadie, J., LIGO and Virgo collaborations.: Predictions for the Rates of Compact Binary Coalescences Observable by Ground-based Gravitational-wave Detectors, arXiv:1003.2480, Class. Quantam Grav. (2010) (submitted)

9. The Virgo Collaboration, Advanced Virgo Preliminary Design, VIRu089A08 (2009)

10. The Einstein Telescope design study (FP7-Capacities, Grant Agreement 211743), http://www.et-gw. eu/

11. Hough, J., Rowan, S.: J. Opt. A: Pure Appl. Opt. 7, S257-S264 (2005)

12. Bender, P.L., Danzmann, K.: (the LISA Study Team) Laser interferometer space antenna for the detection of gravitational waves. Pre-Phase A Report, Doc. MPQ 233 Max-Planck-Institüt für Quantenoptik, Garching (1998)

13. Shaddock, D.A.: Class. Quantum Grav. 25, 114012 (2008)

14. Braccini, S., et al.: Astroparticle Phys. 23, 557-565 (2005)

15. Acernese, F., et al.: Measurements of superattenuator seismic isolation by virgo interferometer. Astropart. Phys. 33(3):182-189 (2010)

16. Abbott, R., et al.: Class. Quantum Grav. 19, 1591-1597 (2002)

17. Bialowons, W., et al.: Measurement of ground motion in various sites, EUROTeV-Report-2007-011 (2007)

18. Uchiyama, T., et al.: Class. Quantum Grav. 21, S1161 (2004)

19. Ando, M., et al.: Phys. Rev. Lett. 86, 3950 (2001)

20. Sato, S., et al.: Phys. Rev. D 69, 102005 (2004)

21. Saulson, P.R.: Phys. Rev. D 30, 732-736 (1984)

22. Beccaria, M.: Class. Quantum Grav. 15, 3339-3362 (1998)

23. Hughes, S.A., Thorne, K.S.: Phys. Rev. D 58, 122002 (1998)

24. Cella, G.: Low Frequency Limit. Talk at the Fujihara Seminar, http://gw.icrr.u-tokyo.ac.jp:8888/ fujihara_seminar_presentation/presentations/Fujihara2009-Cella.pdf (2009)

25. Cella, G.: Gravity Gradient Noise: estimates \& reduction strategies, Talk at the 2nd ET Annual meeting, http://www.et-gw.eu/2ndgeneralworkshop (2009)

26. Callen, H.B., Welton, T.A.: Phys. Rev. 83, 34 (1951)

27. Kovalik, J., Saulson, P.R.: Rev. Sci. Instrum. 64, 2942 (1993)

28. Braginsky, V.B., et al.: Phys. Lett. A 175, 82 (1993)

29. Rowan, S., et al.: Phys. Lett. A 227, 153 (1997)

30. Cagnoli, G., et al.: Phys. Lett. A 255, 230-235 (1999)

31. Cagnoli, G., et al.: Phys. Lett. A 213, 245-252 (1996)

32. Cagnoli, G., et al.: Rev. Scientific Instrum. 71, 2206-2210 (2000)

33. Robertson, N., et al.: In: Proceedings of the Third E. Amaldi Conference AIP, New York, 313 (2000)

34. Cagnoli, G., et al.: Phys. Rev. Lett. 85, 2442-2445 (2000)

35. Rowan, S., et al.: Phys. Lett. A 246, 471-478 (1998)

36. Cagnoli, G., et al.: Rev. Scientific Instrum. 73, 3318-3323 (2002)

37. Kuroda, K., et al.: Int. J. Modern Phys. D 8, 557-579 (1999)

38. Puppo, P., Ricci, F.: Gen. Relativ. Gravit. (2009, in preparation)

39. Caparrelli, S., et al.: Report ILIAS-JR3-C1 activity, http://www.ego-gw.it/ILIAS-GW/documents/ STREGAreport2007/Long reports/Report_C1_2007_D10.doc (2007)

40. Uchiyama, T., et al.: Phys. Lett. A 273, 310-315 (2000)

41. Tomaru, T., et al.: Phys. Lett. A 310, 215-219 (2002)

42. Alshourbagy, M., et al.: Rev. Scientific Instrum. 77, 044502 (2006)

43. Reid, S., et al.: Phys. Lett. A 351, 205-211 (2006)

44. Crooks, D.R.M., et al.: Class. Quantum Grav. 21, S1059-S1065 (2004) 
45. Harry, G.M., et al.: Class. Quantum Grav. 19, 897-917 (2002)

46. Harry, G.M., et al.: Class. Quantum Grav. 24, 405-415 (2007)

47. Agresti, J., et al.: Optimized multilayer dielectric mirror coatings for gravitational wave interferometers. In: Advances in thin-film coatings for optical applications, Proceedings of SPIE, 6286, pp. 628608.1628608.10 (2006) (LIGO P060027-00-Z)

48. Yamamoto, K., et al.: Phys. Rev. D 74, 022002 (2006)

49. Martin, I., et al.: Class. Quantum Grav. 25, 055005 (2008)

50. Villar, A.E., et al.: Measurement of Thermal Noise in Multilayer Coatings with Optimized Layer Thickness (in preparation)

51. Tomaru, T., et al.: Class. Quantum Grav. 19, 2045 (2002)

52. Tomaru, T., et al.: Phys. Lett. A 283, 80-84 (2001)

53. Green, M.A., Keevers, M.J.: Progr. Photov. Res. Appl. 3, 189 (1995)

54. Brückner, F., et al.: Optics Express 17, 163-169 (2009)

55. Bunkowski, A., et al.: J. Phys. Conf. Ser. 32, 333-338 (2006)

56. Franc, J., et al.: ET internal note: ET-021-09 (2009)

57. Agresti, J., De Salvo, R.: Flat Beam Profile to Depress Thermal Noise, LIGO-G050041-00-Z (2005)

58. Mours, B., et al.: Class. Quantum Grav. 23, 5777-5784 (2006)

59. Franc, J., et al.: Effect of Laguerre Gauss modes on thermal noise, talk at the ET-WP3-meeting, https:// workarea.et-.gw.eu/et/WG3-.Topology/presentations/Franc-WP3-090609.ppt (2009)

60. Zeilinger, A.: General properties of lossless beam splitters in interferometry. Am. J. Phys. 49(9) (1981)

61. Corbitt, T.R.: Quantum noise and radiation pressure effects in high power optical interferometers. $\mathrm{PhD}$ Thesis, MIT (2008)

62. http://www.ioffe.rssi.ru/SVA/NSM/Semicond/Si/thermal.html

63. Okada, Y., Tokumaru, Y.: J. Appl. Phys. 56, 2314 (1984)

64. Damon, D.H.: Phys. Rev. B 8, 5860 (1973)

65. Glassbrenner, C.J., Slack, G.A.: Phys. Rev. 134, A1058-A1069 (1964)

66. Nawrodt, R., et al.: J. Phys. Conf. Ser. 122, 012008 (2008)

67. Mavalvala, N., McClelland, D.E., Mueller, G., Reitze, D.H., Schnabel, R., Willke, B.: Gen. Relativ. Gravit. (2009, in preparation)

68. Buonanno, A., Chen, Y.: Phys. Rev. D 67, 062002 (2003)

69. Buonanno, A., Chen, Y.: Phys. Rev. D 69, 102004 (2004)

70. Kimble, H.J., Levin, Y., Matsko, A.B., Thorne, K.S., Vyatchanin, S.P. (KLMTV): PRD 65, 022002 (2001)

71. Corbitt, T., et al.: Optical Cavities as amplitude filter of squeezed fields. Phys. Rev. D 70, 022002 (2004)

72. Khalili, F.Y., et al.: ArXiv:0905.1291v2:[gr-qc] (2009)

73. Thüring, A., et al.: Opt. Lett. 34, 825-826 (2009)

74. Willke, B., et al.: Class. Quantum Grav. 23, S207-S214 (2006)

75. Braginsky, V.B., et al.: Phys. Lett. A 287, 331 (2001)

76. Ju, L., et al.: Phys. Lett. A 354, 360 (2006)

77. Ju, L., et al.: Phys. Lett. A 355, 419 (2006)

78. Yamamoto, K., et al.: J. Phys. Conf. Ser. 122, 012015 (2008)

79. Yamamoto, K.: Parametric instability of a cavity of Einstein Telescope, ET-029-09 (2009)

80. Dimopoulos, S., et al.: Gravitational Wave Detection with Atom Interferometry. arXiv:0712.1250v1 [gr-qc]

81. Chen, Y., Danilishin, S.L., Khalili, F.Ya., Müller-Ebhardt, H.: Gen. Relativ. Gravit. (2009, in preparation)

82. Hild, S., et al.: Pushing towards the ET sensitivity using conventional technology (2008). arXiv:0810.0604v2 [gr-qc]

83. Shoemaker, D.: presentation at Aspen meeting, http://www.ligo.caltech.edu/docs/G/G010026-00.pdf (2001)

84. Conforto, G., DeSalvo, R.: Nucl. Instr. Methods Phys. Res. Sect. A 518, 228-232 (2004)

85. Hild, S., et al.: A Xylophone Configuration for a third Generation Gravitational Wave Detector (2009), submitted to CQG. arXiv:0906.2655v2 [gr-qc]

86. Winkler, W., et al.: Plans for a large gravitational wave antenna in Germany MPQ Report 101 (presented by A Rüdiger at the 4th Marcel Grossmann Meeting, Rome) (1985)

87. Maischberger K., et al.: Vorschlag zum Bau eines groen Laser-Interferometers zur Messung von Gravitationswellen MPQ Report 96 (in German) (1985) 
88. Freise, A., et al.: Class. Quantum Grav. 26, 085012 (2009)

89. Nayak, K.R., et al.: Phys. Rev. D 68, 122001 (2003)

90. Nayak, K.R., et al.: Phys. Rev. D 70, 049901 (2004)

91. Sathyaprakash, B.S., Schutz, B.F.: Living Review in Relativity, Physics, Astrophysics and Cosmology with Gravitational Waves, (http://relativity.livingreviews.org/open?pubNo=lrr-2009-2\& page=articlesu30.html)

92. McKechan, D., et al.: Gravitational Waves with Amplitude Corrections and Higher Harmonics from Compact Binary Coalescences, LIGO-G0900318-v3 (2009)

93. Amaro-Seoane, P., et al.: Einstein telescope design study: vision document. (2009, in preparation) (https://workarea.et-gw.eu/et/WG4-Astrophysics/visdoc/VisDoc-090616.pdf) 